\title{
The existence and multiplicity of positive solutions of nonlinear sixth-order boundary value problem with three variable coefficients
}

Wanjun Li

Correspondence: Iwj1965@163.com Department of Mathematics, Longdong University, Qingyang 745000, Gansu, P. R. China

\section{Abstract}

In this article, we discuss the existence and multiplicity of positive solutions for the sixth-order boundary value problem with three variable parameters as follows:

$$
\left\{\begin{array}{l}
u^{(6)}+A(t) u^{(4)}+B(t) u^{(2)}+C(t) u+f(x, u)=0 \\
u(0)=u(1)=u^{\prime \prime}(0)=u^{\prime \prime}(1)=u^{(4)}(0)=u^{(4)}(1)=0
\end{array}\right.
$$

where $A(t), B(t), C(t) \in C[0,1], f(t, u):[0,1] \times[0, \infty) \rightarrow[0 . \infty)$ is continuous. The proof of our main result is based upon spectral theory of operators and fixed point theorem in cone.

Keywords: sixth-order differential equation, positive solution, fixed point theorem, spectral theory of operators

\section{Introduction}

In this article, we study the existence and multiplicity of positive solution for the following nonlinear sixth-order boundary value problem (BVP for short) with three variable parameters

$$
\left\{\begin{array}{c}
-u^{(6)}-C(t) u^{(4)}+B(t) u^{\prime \prime}-A(t) u=f(t, u), \quad t \in(0,1) \\
u(0)=u(1)=u^{\prime \prime}(0)=u^{\prime \prime}(1)=u^{(4)}(0)=u^{(4)}(1)=0
\end{array}\right.
$$

where $A(t), B(t), C(t) \in C[0,1], f(t, u):[0,1] \times[0, \infty) \rightarrow[0 . \infty)$ is continuous.

In recent years, BVPs for sixth-order ordinary differential equations have been studied extensively, see [1-7] and the references therein. For example, Tersian and Chaparova [1] have studied the existence of positive solutions for the following systems (1.2):

$$
\left\{\begin{array}{l}
u^{(6)}+A u^{(4)}+B u^{\prime \prime}+C u-f(t, u)=0 . \quad 0<x<L, \\
u(0)=u(L)=u^{\prime \prime}(0)=u^{\prime \prime}(L)=u^{(4)}(0)=u^{(4)}(L)=0,
\end{array}\right.
$$

where $A, B$, and $C$ are some given real constants and $f(x, u)$ is a continuous function on $\mathbf{R}^{2}$, is motivated by the study for stationary solutions of the sixth-order parabolic differential equations 


$$
\frac{\partial u}{\partial t}=\frac{\partial^{6} u}{\partial x^{6}}+A \frac{\partial^{4} u}{\partial x^{4}}+B \frac{\partial^{2} u}{\partial x^{2}}+f(x, u)
$$

This equation arose in the formation of the spatial periodic patterns in bistable systems and is also a model for describing the behaviour of phase fronts in materials that are undergoing a transition between the liquid and solid state. When $f(x, u)=u-u^{3}$, it was studied by Gardner and Jones [2] as well as by Caginalp and Fife [3]. In [1], existence of nontrivial solutions for (1.2) is proved using a minimization theorem and a multiplicity result using Clarks theorem when $C=1$ and $f(x, u)=u^{3}$. The authors have studied also the homoclinic solutions for (1.2) when $C=-1$ and $f(x, u)=-a(x) u|u|^{\sigma}$, where $a(x)$ is a positive periodic function and $\sigma$ is a positive constant by the mountain-pass theorem of Brezis-Nirenberg and concentration-compactness arguments. In [4], by variational tools, including two Brezis-Nirenbergs linking theorems, Gyulov et al. have studied the existence and multiplicity of nontrivial solutions of BVP (1.2).

Recently, in [5], the existence and multiplicity of positive solutions of sixth-order BVP with three parameters

$$
\left\{\begin{array}{l}
-u^{(6)}-\gamma u^{(4)}+\beta u^{\prime \prime}-\alpha u=f(t, u), \quad t \in[0,1] \\
u^{(i)}(0)=u^{(i)}(1)=0, \quad i=0,1,2,3,4,5
\end{array}\right.
$$

has been studied under the hypothesis of

$\left(A_{1}\right) f:[0,1] \times[0, \infty) \rightarrow[0, \infty)$ is continuous.

$\left(A_{2}\right) \alpha, \beta, \gamma \in \mathbf{R}$ and under the condition of satisfying

$$
\begin{aligned}
& \frac{\alpha}{\pi^{6}}+\frac{\beta}{\pi^{4}}+\frac{\gamma}{\pi^{2}}<1, \\
& 3 \pi^{4}-2 \gamma \pi^{2}-\beta>0, \quad \gamma<3 \pi^{2} \\
& 18 \alpha \beta \gamma-\beta^{2} \gamma^{2}+4 \alpha \gamma^{3}+27 \alpha^{2}-4 \beta^{3} \leq 0
\end{aligned}
$$

the existence and multiplicity for positive solution of BVP (1.3) are established by using fixed point index theory. In this article, we consider more general BVP (1.1), based upon spectral theory of operators and fixed point theorem in cone, we will establish the existence and multiplicity positive solution of BVP (1.1) and extend the result of [5] under appropriate conditions. Our ideas mainly come from [5,8-10].

We list the following conditions for convenience:

$\left(H_{1}\right) f:[0,1] \times[0,+\infty) \rightarrow[0,+\infty)$ is continuous.

$\left(H_{2}\right) A(t), B(t), C(t) \in C[0,1], \alpha=\min _{0 \leq t \leq 1} \mathrm{~A}(t), \beta=\min _{0 \leq t \leq 1} B(t), \gamma=\min _{0 \leq t \leq 1} C(t)$, and satisfies

$$
\begin{aligned}
& \frac{\alpha}{\pi^{6}}+\frac{\beta}{\pi^{4}}+\frac{\gamma}{\pi^{2}}<1, \\
& 3 \pi^{4}-2 \gamma \pi^{2}-\beta>0, \quad \gamma<3 \pi^{2} \\
& 18 \alpha \beta \gamma-\beta^{2} \gamma^{2}+4 \alpha \gamma^{3}+27 \alpha^{2}-4 \beta^{3} \leq 0 .
\end{aligned}
$$

Let $Y=C[0,1], Y_{+}=\{u \in Y: u(t) \geq 0, t \in[0,1]\}$. It is well known that $Y$ is a Banach space equipped with the norm $\|u\|_{0}=\sup _{0 \leq t \leq 1}|u(t)|, u \in Y$. Set $X=\left\{u \in C^{4}[0,1]: u\right.$ $\left.(0)=u(1)=u^{\prime \prime}(0)=u^{\prime \prime}(1)=0\right\}$, then $X$ also is a Banach space equipped with the norm $\|u\|_{X}=\max \left\{\|u(t)\|_{0},\left\|u^{\prime \prime}(t)\right\|_{0},\left\|u^{(4)}(t)\right\|_{0}\right\}$. If $u \in C^{4}[0,1] \cap C^{6}(0,1)$ fulfils BVP (1.1), 
then we call $u$ is a solution of BVP (1.1). If $u$ is a solution of BVP (1.1), and $u(t)>0, t$ $\in(0,1)$, then we say $u$ is a positive solution of BVP $(1.1)$.

\section{Preliminaries}

In this section, we will make some preliminaries which are needed to show our main results.

Lemma 2.1. Let $u \in X$, then $\|u\|_{0} \leq\left\|u^{\prime \prime}\right\|_{0} \leq\left\|u^{(4)}\right\|_{0} \leq\|u\|_{X}$.

Proof. The proof is similar to the Lemma 1 in [8], so we omit it. $\square$

Lemma 2.2. [5] Let $\lambda_{1}, \lambda_{2}$, and $\lambda_{3}$ be the roots of the polynomial $P(\lambda)=\lambda^{3}+\gamma \lambda^{2}$ $\beta \lambda+\alpha$. Suppose that condition $\left(H_{2}\right)$ holds, then $\lambda_{1}, \lambda_{2}$, and $\lambda_{3}$ are real and greater than $-\pi^{2}$.

Note : Based on Lemma 2.2, it is easy to learn that when the three parameters satisfy the condition of $\left(H_{2}\right)$, they satisfy the condition of non-resonance.

Let $G_{i}(t, s)(i=1,2,3)$ be the Green's function of the linear BVP

$-u^{\prime \prime}(t)+\lambda_{i} u(t)=0, u(0)=u(1)=0$,

Lemma 2.3. [10] $G_{i}(t, s)(i=1,2,3)$ has the following properties

(c1) $G_{i}(t, s)>0, \forall t, s \in(0,1)$.

(c2) $G_{i}(t, s)<C_{i} G_{i}(s, s), \forall t, s \in[0,1]$, in which $C_{i}>0$ is constant.

(c3) $G_{i}(t, s) \geq \delta_{i} G_{i}(t, t) G_{i}(s, s), \forall t, s \in[0,1]$, in which $\delta_{i}>0$ is constant.

We set

$$
\begin{aligned}
M_{i}=\max _{0 \leq t \leq 1} G_{i}(s, s), \quad m_{i}=\min _{\frac{1}{4} \leq t \leq \frac{3}{4}} G_{i}(s, s), \quad i=1,2,3 . \\
C_{i j}=\int_{0}^{1} G_{i}(\delta, \delta) G_{j}(\delta, \delta) d \delta, \quad c_{i j}=\int_{\frac{1}{4}}^{\frac{3}{4}} G_{i}(\delta, \delta) G_{j}(\delta, \delta) d \delta, \quad i, j=1,2,3 . \\
D_{i}=\max _{0 \leq t \leq 1} \int_{0}^{1} G_{i}(t, s) d s, \quad d_{i}=\max _{\frac{1}{4} \leq t \leq \frac{3}{4}} \frac{1}{4} G_{i}(t, s) d s \quad i=1,2,3,
\end{aligned}
$$

then starting from Lemma 2.3 we know $M_{i}, m_{i}, C_{i j}>0$.

For any $h \in Y$, take into consideration of linear BVP:

$$
\left\{\begin{array}{l}
-u^{(6)}-\gamma u^{(4)}+\beta u^{\prime \prime}-\alpha u=h(t), \quad t \in[0,1] \\
u(0)=u(1)=u^{\prime \prime}(0)=u^{\prime \prime}(1)=u^{(4)}(0)=u^{(4)}(1)=0,
\end{array}\right.
$$

where $\alpha, \beta, \gamma$ satisfy assumption $\left(H_{2}\right)$. Since

$$
-u^{(6)}-\gamma u^{(4)}+\beta u^{\prime \prime}-\alpha u=\left(-\frac{d^{2}}{d t^{2}}+\lambda_{1}\right)\left(-\frac{d^{2}}{d t^{2}}+\lambda_{2}\right)\left(-\frac{d^{2}}{d t^{2}}+\lambda_{3}\right) u,
$$

then for any $h \in Y$, the $\operatorname{LBVP}(2.4)$ has a unique solution $u$, which we denoted by $A h$ $=u$. The operator $A$ can be expressed by 


$$
u(t)=(A h)(t):=\int_{0}^{1} \int_{0}^{1} \int_{0}^{1} G_{1}(t, \delta) G_{2}(\delta, \tau) G_{3}(\tau, s) h(t) d s d \tau d \delta
$$

Lemma 2.4. The linear operator $A: Y \rightarrow X$ is completely continuous and $\|A\| \leq \varpi$, where $\omega=\left|\lambda_{2}+\lambda_{3}\right|\left(C_{1} C_{2} C_{3} M_{1} M_{2} M_{3}\left|\lambda_{3}\right|+C_{1} C_{2} M_{1} M_{2}\right)+\left|\lambda_{2} \lambda_{3}\right|\left(C_{1} C_{2} C_{3} M_{1} M_{2} M_{3}+C_{1} M_{1}\right)$.

Proof. It is easy to show that the operator $A: Y \rightarrow X$ is linear operator. $\forall h \in Y, u=$ $A h \in X, u(0)=u(1)=u^{\prime \prime}(0)=u^{\prime \prime}(1)=u^{(4)}(0)=u^{(4)}(1)=0$. Let $v=\left(-\frac{d^{2}}{d t^{2}}+\lambda_{2}\right)\left(-\frac{d^{2}}{d t^{2}}+\lambda_{3}\right) u$, that is

$$
v=\left(-\frac{d^{2}}{d t^{2}}+\lambda_{2}\right)\left(-\frac{d^{2}}{d t^{2}}+\lambda_{3}\right) u=u^{(4)}-\left(\lambda_{2}+\lambda_{3}\right) u^{\prime \prime}+\lambda_{2} \lambda_{3} u,
$$

by (2.5) and (2.7), we have

$$
\left\{\begin{array}{l}
-v^{\prime \prime}+\lambda_{1} v=h(t), \quad t \in(0,1) \\
v(0)=v(1)=0,
\end{array}\right.
$$

and $v(t)=\int_{0}^{1} G_{1}(t, s) h(s) d s, t \in[0,1]$, so

$$
u^{(4)}-\left(\lambda_{2}+\lambda_{3}\right) u^{\prime \prime}+\lambda_{2} \lambda_{3} u=\int_{0}^{1} G_{1}(t, s) h(s) d s, \quad t \in[0,1] .
$$

By (2.6), for any $t \in[0,1]$, we have

$$
|u(t)| \leq \int_{0}^{1} \int_{0}^{1} \int_{0}^{1} G_{1}(t, \delta) G_{2}(\delta, \tau) G_{3}(\tau, s)|h(t)| d s d \tau d \delta \leq C_{1} C_{2} C_{3} M_{1} M_{2} M_{3}\|h\|_{0} .
$$

Again, let $\omega=-u^{\prime \prime}+\lambda_{3} u$, then $\omega(0)=\omega(1)=\omega^{\prime \prime}(0)=\omega^{\prime \prime}(1)=0$, by $(2,5)$, we have

$$
\left\{\begin{array}{l}
\omega^{(4)}-\left(\lambda_{1}+\lambda_{2}\right) \omega^{\prime \prime}+\lambda_{1} \lambda_{2} \omega=h(t), \quad t \in(0,1), \\
\omega(0)=\omega(1)=\omega^{\prime \prime}(0)=\omega^{\prime \prime}(1)=0 .
\end{array}\right.
$$

Then $\omega(t)=\int_{0}^{1} \int_{0}^{1} G_{1}(t, \tau) G_{2}(\tau, s) h(s) d s d \tau, t \in[0,1]$, that is

$$
-u^{\prime \prime}+\lambda_{3} u=\int_{0}^{1} \int_{0}^{1} G_{1}(t, \tau) G_{2}(\tau, s) h(s) d s d \tau, \quad t \in[0,1] .
$$

So

$$
\left|u^{\prime \prime}\right| \leq C_{1} C_{2} M_{1} M_{2}\left(1+\left|\lambda_{3}\right| C_{3} M_{3}\right)\|h\|_{0}, \quad t \in[0,1] .
$$

Based on (2.8), (2.9), and (2.12), we have

$$
\begin{aligned}
\left|u^{(4)}(t)\right| \leq & \left|\lambda_{2}+\lambda_{3}\right|\left|u^{\prime \prime}(t)\right|+\left|\lambda_{2} \lambda_{3}\right||u(t)|+\int_{0}^{1} G_{1}(t, s)|h(s)| d s \\
\leq & \left|\lambda_{2}+\lambda_{3}\right|\left(C_{1} C_{2} C_{3} M_{1} M_{2} M_{3}\left|\lambda_{3}\right|+C_{1} C_{2} M_{1} M_{2}\right)\|h\|_{0} \\
& +\left|\lambda_{2} \lambda_{3}\right|\left(C_{1} C_{2} C_{3} M_{1} M_{2} M_{3}\|h\|_{0}+C_{1} M_{1}\right)\|h\|_{0} \\
\leq & \varpi\|h\|_{0}, \quad t \in[0,1],
\end{aligned}
$$


where

$$
\begin{aligned}
\varpi & =\left|\lambda_{2}+\lambda_{3}\right|\left(C_{1} C_{2} C_{3} M_{1} M_{2} M_{3}\left|\lambda_{3}\right|+C_{1} C_{2} M_{1} M_{2}\right) \\
& +\left|\lambda_{2} \lambda_{3}\right|\left(C_{1} C_{2} C_{3} M_{1} M_{2} M_{3}+C_{1} M_{1}\right) .
\end{aligned}
$$

So, $\left\|u^{(4)}(t)\right\| \leq \varpi\|h\|_{0}$, by Lemma2.1, $\|u\|_{X} \leq \varpi\|h\|_{0}$, then

$$
\|A h\|_{X} \leq \varpi\|h\|_{0},
$$

so $A$ is continuous, and $\|A\| \leq \varpi$.

Next, we will show that $A$ is compact with respect to the norm $\|\cdot\|_{X}$ on $X$.

Suppose $\left\{h_{n}\right\}(n=1,2, \ldots)$ an arbitrary bounded sequence in $Y$, then there exists $K_{0}$ $>0$ such that $\left\|h_{n}\right\|_{0} \leq K_{0}, n=1,2, \ldots$ Let $u_{n}=A h_{n}, 1,2, \ldots$ By $(2.8), \forall t_{1}, t_{2} \in[0,1]$, $t_{1}<t_{2}$, we have

$$
\begin{aligned}
& \left|u_{n}^{(4)}\left(t_{2}\right)-u_{n}^{(4)}\left(t_{1}\right)\right| \\
& \leq\left|\lambda_{2}+\lambda_{3}\right|\left|u_{n}^{\prime \prime}\left(t_{2}\right)-u_{n}^{\prime \prime}{ }_{n}\left(t_{1}\right)\right|+\left|\lambda_{2} \lambda_{3}\right|\left|u_{n}\left(t_{2}\right)-u_{n}\left(t_{1}\right)\right|+\int_{0}^{1}\left|G_{1}\left(t_{2}, s\right)-G_{1}\left(t_{1}, s\right)\right|\left|h_{n}(s)\right| d s . \\
& \leq\left|\lambda_{2}+\lambda_{3}\right|\left(\left|\lambda_{3}\right|\left|u_{n}\left(t_{2}\right)-u_{n}\left(t_{1}\right)\right|+\int_{0}^{1} \int_{0}^{1}\left|G_{1}\left(t_{2}, \tau\right)-G_{1}\left(t_{1}, \tau\right)\right|\left|G_{2}(\tau, s)\right|\left|h_{n}(s)\right| d s d \tau\right) \\
& +\left|\lambda_{2} \lambda_{3}\right|\left|u_{n}\left(t_{2}\right)-u_{n}\left(t_{1}\right)\right|+\int_{0}^{1}\left|G_{1}\left(t_{2}, s\right)-G_{1}\left(t_{1}, s\right)\right|\left|h_{n}(s)\right| d s . \\
& \leq\left(\lambda_{3}^{2}+2\left|\lambda_{2} \lambda_{3}\right|\right) \int_{0}^{1} \int_{0}^{1} \int_{0}^{1}\left|G_{1}\left(t_{2}, \delta\right)-G_{1}\left(t_{1}, \delta\right)\right|\left|G_{2}(\delta, \tau)\right|\left|G_{3}(\tau, s)\right|\left|h_{n}(s)\right| d s d \tau d \delta . \\
& \quad+\left|\lambda_{2}+\lambda_{3}\right| \int_{0}^{1} \int_{0}^{1}\left|G_{1}\left(t_{2}, \tau\right)-G_{1}\left(t_{1}, \tau\right)\right|\left|G_{2}(\tau, s) \| h_{n}(s)\right| d s d \tau \\
& \quad+\int_{0}^{1}\left|G_{1}\left(t_{2}, s\right)-G_{1}\left(t_{1}, s\right)\right|\left|h_{n}(s)\right| d s . \\
& \leq\left(\left(\lambda_{3}^{2}+2\left|\lambda_{2} \lambda_{3}\right|\right) \int_{0}^{1} \int_{0}^{1} \int_{0}^{1}\left|G_{1}\left(t_{2}, \delta\right)-G_{1}\left(t_{1}, \delta\right)\right| G_{2}(\delta, \tau) G_{3}(\tau, s) d s d \tau d \delta .\right. \\
& \left.\quad+\left|\lambda_{2}+\lambda_{3}\right| \int_{0}^{1} \int_{0}^{1}\left|G_{1}\left(t_{2}, \tau\right)-G_{1}\left(t_{1}, \tau\right) G_{2}(\tau, s) d s d \tau+\int_{0}^{1}\right| G_{1}\left(t_{2}, s\right)-G_{1}\left(t_{1}, s\right) \mid d s\right) K_{0} .
\end{aligned}
$$

Because $G_{i}(t, s)(i=1,2,3)$ is uniform continuity on $[0,1] \times[0,1]$, based on the above demonstration, it is easy to proof that $\left\{u_{n}^{(4)}\right\}_{n=1}^{\infty}$ is equicontinuous on $[0,1]$. From (2.15), we know $\|u\|_{0},\left\|u^{\prime \prime}\right\|_{0},\left\|u^{(4)}\right\|_{0} \leq\|u\|_{X} \leq \varpi\left\|h_{n}\right\|_{0} \leq \varpi K_{0}$, so $\left\{u_{n}(t)\right\},\left\{u_{n}^{\prime \prime}(t)\right\}$ and $\left\{u_{n}^{(4)}(t)\right\}$ are relatively compact in $\mathbf{R}$. Based on Lemma 1.2.7 in [11], we know $\left\{u_{n}\right\}_{n=1}^{\infty}$ is the relatively compact in $X$, so $A$ is compact operator. $\square$

The main tools of this article are the following well-known fixed point index theorems.

Let $E$ be a Banach Space and $K \subset E$ be a closed convex cone in $E$. Assume that $\Omega$ is a bounded open subset of $E$ with boundary $\partial \Omega$, and $K \cap \Omega \neq \varnothing$. Let $A: K \cap \bar{\Omega} \rightarrow K$ be a completely continuous mapping. If $A u \neq u$ for every $u \in K \cap \partial \Omega$, then the fixed 
point index $i(A, K \cap \Omega, K)$ is well defined. We have that if $i(A, K \cap \Omega, K) \neq 0$, then $A$ has a fixed point in $K \cap \Omega$.

Let $K_{r}=\{u \in K\|\| u \|<r\}$ and $\partial K_{r}=\{u \in K\|u\|<r\}$ for every $r>0$.

Lemma 2.5. [12] Let $A: K \rightarrow K$ be a completely continuous mapping. If $\mu A u \neq u$ for every $u \in \partial K_{r}$ and $0<\mu \leq 1$, then $i\left(A, K_{r}, K\right)=1$.

Lemma 2.6. [12] Let $A: K \rightarrow K$ be a completely continuous mapping. Suppose that the following two conditions are satisfied:

(i) $\inf _{u \in \partial K_{r}}|| A u \|>0$,

(ii) $\mu A u \neq u$ for every $u \in \partial K_{r}$ and $\mu \geq 1$,

then $i\left(A, K_{r}, K\right)=0$.

Lemma 2.7. [12] Let $X$ be a Banach space, and let $K \subseteq X$ be a cone in $X$. For $p>0$, define $K_{p}=\{u \in K \mid\|u\|<p\}$. Assume that $A: K_{p} \rightarrow K$ is a completely continuous mapping such that $A u \neq u$ for every $u \in \partial K_{p}=\{u \in K \mid\|u\|=p\}$.

(i) If $\|u\| \leq\|A u\|$, for every $u \in \partial K_{p}$, then $i\left(A, K_{p}, K\right)=0$.

(ii) If $\|u\| \geq\|A u\|$, for every $u \in \partial K_{p}$, then $i\left(A, K_{p}, K\right)=1$.

\section{Main results}

We bring in following notations in this section:

$$
\begin{aligned}
& \underline{f}_{0}=\lim _{u \rightarrow 0_{+}} \inf \min _{0 \leq t \leq 1}(f(t, u) / u), \quad \bar{f}_{\infty}=\lim _{u \rightarrow+\infty} \sup \max _{0 \leq t \leq 1}(f(t, u) / u), \\
& \bar{f}_{0}=\lim _{u \rightarrow 0_{+}} \sup \max _{0 \leq t \leq 1}(f(t, u) / u), \quad \underline{-}_{-\infty}=\lim _{u \rightarrow+\infty} \inf \min _{0 \leq t \leq 1}(f(t, u) / u) . \\
& a(t)=A(t)-\alpha, \quad b(t)=B(t)-\beta, \quad c(t)=C(t)-\gamma, \\
& \Gamma=\pi^{6}-\gamma \pi^{4}-\beta \pi^{2}-\alpha, \quad K=\max _{0 \leq t \leq 1}[a(t)+b(t)+c(t)],
\end{aligned}
$$

Suppose that:

$\left(H_{3}\right) L=\varpi K<1$, where $\varpi$ is defined as in (2.14).

Theorem 3.1. Assume that $\left(H_{1}\right)-\left(H_{3}\right)$ hold, and $b(t) \geq\left(\lambda_{2}+\lambda_{3}\right) c(t), \lambda_{3} b(t)-a(t) \leq \lambda_{3}^{2} c(t)$, then in each of the following cases:

(i) $\underline{f}_{-0}>\Gamma, \bar{f}_{\infty}<(1-L) \Gamma$, (ii) $\bar{f}_{0}<(1-L) \Gamma, \underline{f}_{-\infty}>\Gamma$, the BVP (1.1) has at least one positive solution.

Proof. $\forall h \in Y$, consider the LBVP

$$
\left\{\begin{array}{l}
-u^{(6)}-C(t) u^{(4)}+B(t) u^{\prime \prime}-A(t) u=h(t), \\
u(0)=u(1)=u^{\prime \prime}(0)=u^{\prime \prime}(1)=u^{(4)}(0)=u^{(4)}(1)=0, \quad 0<t<1
\end{array}\right.
$$

It is easy to prove (3.1) is equivalent to the following BVP

$$
\left\{\begin{array}{l}
-u^{(6)}-\gamma u^{(4)}+\beta u^{\prime \prime}-\alpha=G u+h(t), \\
u(0)=u(1)=u^{\prime \prime}(0)=u^{\prime \prime}(1)=u^{(4)}(0)=u^{(4)}(1)=0, \quad 0<t<1
\end{array}\right.
$$

where $G v:=(C(t)-\gamma) v^{(4)}-(B(t)-\beta) v^{\prime \prime}+(A(t)-\alpha) v, \forall v \in X$. Obviously, the operator $G: X \rightarrow Y$ is linear, and $\forall v \in X, t \in[0,1]$, we have $|G v(t)| \leq K\|v\|_{X}$. Hence $\|G v\|_{0} \leq$ $K\|v\|_{X}$, and so $\|G\| \leq K$. On the other hand, $u \in C^{4}[0,1] \cap C^{6}(0,1), t \in[0,1]$ is a solution of (3.2) iff $u \in X$ satisfies $u=A(G u+h)$, i.e.,

$$
u \in X, \quad(I-A G) u=A h .
$$


Owing to $G: X \rightarrow Y$ and $A: Y \rightarrow X$, the operator $I-A G$ maps $X$ into $Y$. From $A \leq$ $\varpi$ (by Lemma 2.4) together with $\|G\| \leq K$ and condition $\left(H_{3}\right)$, applying operator spectral theorem, we have that the operator $(I-A G)^{-1}$ exists and is bounded. Let $H=(I-$ $A G)^{-1} A$, then (3.3) is equivalent to $u=H h$. By the Neumann expansion formula, $H$ can be expressed by

$$
H=\left(I+A G+\cdots+(A G)^{n}+\cdots\right) A=A+(A G) A+\cdots+(A G)^{n} A+\cdots
$$

The complete continuity of $A$ with the continuity of $(I-A G)^{-1}$ yields that the operator $H: Y \rightarrow X$ is completely continuous. If we restrict $H: Y_{+} \rightarrow Y, \forall h \in Y_{+}$and mark $u=A h$, then $u \in X \cap Y_{+}$. Based on equation (2.8), (2.11) and Lemma 2.4, we have

$$
\begin{aligned}
& u^{\prime \prime}=\lambda_{3} u-\int_{0}^{1} \int_{0}^{1} G_{1}(t, \tau) G_{2}(\tau, s) h(s) d s d \tau \leq \lambda_{3} u, \quad t \in[0,1], \\
& u^{(4)}=\left(\lambda_{2}+\lambda_{3}\right) u^{\prime \prime}-\lambda_{2} \lambda_{3} u+\int_{0}^{1} G_{1}(t, s) h(s) d s \geq\left(\lambda_{2}+\lambda_{3}\right) u^{\prime \prime}-\lambda_{2} \lambda_{3} u, \quad t \in[0,1],
\end{aligned}
$$

by $b(t) \geq\left(\lambda_{2}+\lambda_{3}\right) c(t)$ and $\lambda_{3} b(t)-a(t) \leq \lambda_{3}^{2} c(t)$, we have

$$
\begin{aligned}
(G u)(t) & =c(t) u^{(4)}-b(t) u^{\prime \prime}+a(t) u \\
& \geq\left[\left(\lambda_{2}+\lambda_{3}\right) c(t)-b(t)\right] u^{\prime \prime}-\left[\lambda_{2} \lambda_{3} c(t)-a(t)\right] u \\
& \geq \lambda_{3}\left[\left(\lambda_{2}+\lambda_{3}\right) c(t)-b(t)\right] u-\left[\lambda_{2} \lambda_{3} c(t)-a(t)\right] u \\
& \geq\left[\lambda_{3}^{2} c(t)-\lambda_{3} b(t)+a(t)\right] u \geq 0, \quad t \in[0,1]
\end{aligned}
$$

Hence

$$
\forall h \in Y_{+}, \quad(G A h)(t) \geq 0, \quad \forall t \in[0,1],
$$

and so $(A G)(A h)(t)=A(G A h)(t) \geq 0, \forall t \in[0,1]$. Suppose that $\forall h \in Y_{+},(A G)^{k}(A h)(t)$ $\geq 0, \forall t \in[0,1]$. For any $h \in Y_{+}$, let $h_{1}=G A h$, by (3.5) we have $h_{1} \in Y_{+}$, and so

$$
(A G)^{k+1}(A h)(t)=(A G)^{k}(A G A h)(t)=(A G)^{k}\left(A h_{1}\right)(t) \geq 0, \quad \forall t \in[0,1] .
$$

Thus by induction it follows that $\forall n \geq 1, \forall h \in Y_{+},(A G)^{n}(A h)(t) \geq 0, \forall t \in[0,1]$. By (3.4), we have

$$
\begin{aligned}
\forall h \in Y_{+,}(H h)(t) & =(A h)(t)+(A G)(A h)(t)+\cdots+(A G)^{n}(A h)(t)+\cdots \\
& \geq(A h)(t), \quad \forall t \in[0,1] .
\end{aligned}
$$

So $H: Y_{+} \rightarrow Y_{+} \cap X$.

On the other hand, we have

$$
\begin{aligned}
\forall h \in Y_{+},(H h)(t) & \leq(A h)(t)+\|(A G)\|(A h)(t)+\cdots+\left\|(A G)^{n}\right\|(A h)(t)+\cdots \\
& \leq\left(1+L+\cdots+L^{n}+\cdots\right)(A h)(t) \\
& \leq \frac{1}{1-L}(A h)(t), \quad \forall t \in[0,1]
\end{aligned}
$$


So the following inequalities hold

$$
\|H h\|_{0} \leq \frac{1}{1-L}\|A h\|_{0}, \quad \forall t \in[0,1]
$$

For any $u \in Y_{+}$, define $F u=f(t, u)$. Based on condition $\left(H_{1}\right)$, it is easy to show $F$ : $Y_{+} \rightarrow Y_{+}$is continuous. By (3.1)-(3.3), It is easy to see that $u \in C^{4}[0,1] \cap C^{6}(0,1)$ is a positive solution of BVP (1.1) iff $u \in Y_{+}$is a nonzero solution of an operator equation as follows

$$
u=H F u \text {. }
$$

Let $Q=H F$. Obviously, $Q: Y_{+} \rightarrow Y_{+}$is completely continuous. We next show that the operator $Q$ has at least one nonzero fixed point in $Y_{+}$.

Let

$$
P=\left\{u \in Y_{+} \mid u(t) \geq \sigma\|u\|_{0}, \quad \forall t \in[0,1]\right\}
$$

In which

$$
\sigma=\frac{\delta_{1} \delta_{2} \delta_{3} C_{12} C_{23}}{C_{1} C_{2} C_{3} M_{1} M_{2}}(1-L) G_{1}(t, t)
$$

Here $M_{1}$ and $M_{2}$ can be defined as that in (2.1), $C_{12}$ and $C_{23}$ can be defined as that in (2.2), $C_{i}, \delta_{i}(i=1,2,3)$ can be defined as that in Lemma 2.3. It is easy to prove that $P$ is a cone in $Y$. We will prove $Q P \subset P$ next.

For any $u \in P$, let $h=F u$, then $h \in Y_{+}$. By (3.6) and Lemma 2.3, we have

$$
(\mathrm{Qu})(t)=(H F u)(t) \geq(A F u)(t), \quad \forall t \in[0,1]\}
$$

By Lemma 2.3, for all $u \in P$, we have

$$
\begin{aligned}
(A F u)(t) & =\int_{0}^{1} \int_{0}^{1} \int_{0}^{1} G_{1}(t, \delta) G_{2}(\delta, \tau) G_{3}(\tau, s)(F u)(s) d s d \tau d \delta \\
& \leq C_{1} C_{2} C_{3} M_{1} M_{2} \int_{0}^{1} G_{3}(s, s)(F u)(s) d s .
\end{aligned}
$$

And accordingly we have $\|A F u\|_{0} \leq C_{1} C_{2} C_{3} M_{1} M_{2} \int_{0}^{1} G_{3}(s, s)(F u)(s) d s$, that is

$$
\int_{0}^{1} G_{3}(s, s)(F u)(s) d s \geq \frac{\|A F u\|_{0}}{C_{1} C_{2} C_{3} M_{1} M_{2}} .
$$


By using (c3) in Lemma 2.3, (3.8) and (3.11), we have

$$
\begin{aligned}
(A F u)(t) & \geq \delta_{1} \delta_{2} \delta_{3} C_{12} C_{23} G_{1}(t, t) \int_{0}^{1} G_{3}(s, s)(F u)(s) d s \\
& \geq \frac{\delta_{1} \delta_{2} \delta_{3} C_{12} C_{23} G_{1}(t, t)}{C_{1} C_{2} C_{3} M_{1} M_{2}}\|A F u\|_{0} \\
& \geq \frac{\delta_{1} \delta_{2} \delta_{3} C_{12} C_{23} G_{1}(t, t)}{C_{1} C_{2} C_{3} M_{1} M_{2}}(1-L)\|H F u\|_{0} \\
& \geq \frac{\delta_{1} \delta_{2} \delta_{3} C_{12} C_{23} G_{1}(t, t)}{C_{1} C_{2} C_{3} M_{1} M_{2}}(1-L)\|Q u\|_{0} .
\end{aligned}
$$

$\operatorname{So}(Q u)(t) \geq \frac{\delta_{1} \delta_{2} \delta_{3} C_{12} C_{23} G_{1}(t, t)}{C_{1} C_{2} C_{3} M_{1} M_{2}}(1-L)\|Q u\|_{0}=\sigma\|Q u\|_{0}$. Thus $Q P \subset P$.

Let

$$
\rho=\frac{\delta_{1} \delta_{2} \delta_{3} C_{12} C_{23} m_{1}(1-L)}{C_{1} C_{2} C_{3} M_{1} M_{2}}
$$

in which $m_{1}$ can be defined as that in (2.1). It's easy to prove

$$
\forall u \in P \Rightarrow u(t) \geq \rho\|u\|_{0}, \quad \forall t \in\left[\frac{1}{4}, \frac{3}{4}\right]
$$

Case (i), since $\underline{f}_{0}>\Gamma$, there exist $\varepsilon>0$ and $r_{0}>0$ such that $f(t, x) \geq(\Gamma+\varepsilon) x, 0 \leq t \leq$ $1,0<x \leq r_{0}$. Let $r \in\left(0, r_{0}\right)$ and $\Omega_{r}=\left\{u \in P \mid\|u\|_{0} \leq r\right\}$, then for every $u \in \partial \Omega_{r}$, we have $\|u\|_{0}=r, 0<u(t) \leq r, t \in(0,1)$, and so $f(t, u(t)) \geq(\Gamma+\varepsilon) u(t), t \in(0,1)$. By (3.13), it follows that

$$
f(t, u(t))>(\Gamma+\varepsilon) u(t) \geq(\Gamma+\varepsilon) \rho r, \quad \forall t \in\left[\frac{1}{4}, \frac{3}{4}\right] .
$$

From (3.6) and (3.14), we have

$$
\begin{aligned}
\|Q u\|_{0} & \geq Q u\left(\frac{1}{2}\right)=(H F u)\left(\frac{1}{2}\right) \geq(A F u)\left(\frac{1}{2}\right) \\
& =\int_{0}^{1} \int_{0}^{1} \int_{0}^{1} G_{1}\left(\frac{1}{2}, \delta\right) G_{2}(\delta, \tau) G_{3}(\tau, s) f(s, u(s)) d s d \tau d \delta \\
& \geq \int_{\frac{1}{4}}^{\frac{3}{4}} \int_{\frac{1}{4}}^{\frac{3}{4}} \int_{\frac{1}{4}}^{\frac{3}{4}} G_{1}\left(\frac{1}{2}, \delta\right) G_{2}(\delta, \tau) G_{3}(\tau, s)(\Gamma+\varepsilon) \rho r d s d \tau d \delta \\
& \geq \delta_{1} \delta_{2} \delta_{3} m_{1} C_{12} C_{23}(\Gamma+\varepsilon) \rho r \int_{\frac{1}{4}}^{\frac{3}{4}} G_{3}(s, s) d s . \\
& \geq \frac{1}{2} \delta_{1} \delta_{2} \delta_{3} m_{1} m_{3} C_{12} C_{23}(\Gamma+\varepsilon) \rho r>0 .
\end{aligned}
$$

Therefore, $\inf _{u \in \partial \Omega_{r}}\|Q u\|_{0}>0$. Now we shall prove $\forall u \in \partial \Omega_{r}, \mu \geq 1, \mu Q u \neq u$. In fact, suppose the contrary, then there exist $u_{0} \in \partial \Omega_{r}$, and $\mu_{0} \geq 1$ such that $\mu_{0} Q u_{0}=u_{0}$. By 
(3.6), we have $u_{0}(t) \geq \frac{1}{\mu_{0}} u_{0}(t)=Q u_{0}(t) \geq\left(A F u_{0}\right)(t)$. Let $\omega_{0}=A F u_{0}$, then $u_{0} \geq \omega_{0}$ and $\omega_{0}(t)$ satisfies BVP (2.4) with $h=F u_{0}$. Hence

$$
\left\{\begin{array}{l}
-\omega_{0}^{(6)}-\gamma \omega_{0}^{(4)}+\beta \omega^{\prime \prime}{ }_{0}-\alpha \omega_{0}=f\left(t, u_{0}\right), \quad t \in(0,1), \\
\omega_{0}(0)=\omega_{0}(1)=\omega^{\prime \prime}{ }_{0}(0)=\omega^{\prime \prime}{ }_{0}(1)=\omega_{0}^{(4)}(0)=\omega_{0}^{(4)}(1)=0,
\end{array}\right.
$$

After multiplying the two sides of the first equation in (3.15) by sin \&\#960t and integrating on $[0,1]$, we have

$$
\Gamma \int_{0}^{1} \omega_{0}(t) \sin \pi t d t=\int_{0}^{1} f\left(t, u_{0}(t)\right) \sin \pi t d t
$$

then

$$
\begin{aligned}
(\Gamma+\varepsilon) \int_{0}^{1} u_{0}(t) \sin \pi t d t & \leq \int_{0}^{1} f\left(t, u_{0}(t)\right) \sin \pi t d t \\
& =\Gamma \int_{0}^{1} \omega_{0}(t) \sin \pi t d t \leq \Gamma \int_{0}^{1} u_{0}(t) \sin \pi t d t .
\end{aligned}
$$

Since $u_{0}(t) \geq \rho\left\|u_{0}\right\|_{0}=\rho r, \forall t \in\left[\frac{1}{4}, \frac{3}{4}\right]$, so $\int_{0}^{1} u_{0}(t) \sin \pi t d t>0$ and we see that $\Gamma$ $+\varepsilon<\Gamma$, which is a contradiction. Then based on Lemma 2.6, we come to

$$
i\left(Q, \Omega_{r}, P\right)=0 .
$$

On the other hand, since $\bar{f}_{\infty}<(1-L) \Gamma$, there exist $\varepsilon \in(0,(1-L) \Gamma)$ and $R_{0}>0$ such that $f(t, x) \leq[(1-L) \Gamma-\varepsilon] x, 0 \leq t \leq 1, x>R_{0}$. Let $M_{R_{0}}=\sup _{0 \leq t \leq 1,0 \leq x \leq R_{0}} f(t, x)$. Then

$$
f(t, x)<[(1-L) \Gamma-\varepsilon] x+M_{R_{0}}, \quad 0 \leq t \leq 1, \quad x \geq 0 .
$$

We choose $R>\max \left\{R_{0}, r, \frac{\sqrt{2} M_{R_{0}}}{\rho \varepsilon}\right\}$ and let $\Omega_{R}=\left\{u \in P\|\| u \|_{0}<R\right\}$. Next we prove $\forall u \in \partial \Omega_{R}, \mu \geq 1, \mu u \neq Q u$. Assume on the contrary that $\exists \mu_{0} \geq 1, u_{0} \in \partial \Omega_{R}$, such that $\mu_{0} u_{0}=Q u_{0}$. Let $\omega_{1}=A F u_{0}$, by (3.6), we have $u_{0} \leq \mu_{0} u_{0}=Q u_{0} \leq \frac{1}{1-L} A F u_{0} \leq \frac{1}{1-L} \omega_{1}$ and $\omega_{1}(t)$ satisfies BVP (2.4) with $h=F u_{0}$. Similarly to (3.16), we can prove

$$
\begin{aligned}
(1-L) \Gamma \int_{0}^{1} u_{0}(t) \sin \pi t d t & \leq \Gamma \int_{0}^{1} \omega_{1}(t) \sin \pi t d t=\int_{0}^{1} f\left(t, u_{0}(t)\right) \sin \pi t d t \\
& \leq[(1-L) \Gamma-\varepsilon] \int_{0}^{1} u_{0}(t) \sin \pi t d t+M_{R_{0}} \int_{0}^{1} \sin \pi t d t
\end{aligned}
$$

and so

$$
\begin{aligned}
M_{R_{0}} \int_{0}^{1} \sin \pi t d t & \geq \varepsilon \int_{0}^{1} u_{0}(t) \sin \pi t d t \geq \varepsilon \int_{\frac{1}{4}}^{\frac{3}{4}} u_{0}(t) \sin \pi t d t \\
& \geq \rho \varepsilon\left\|u_{0}\right\|_{0} \int_{0}^{1} \sin \pi t d t
\end{aligned}
$$


Thus, by (3.19), we have $R=\left\|u_{0}\right\|_{0} \leq \frac{\sqrt{2} M_{R_{0}}}{\rho \varepsilon}$ which is contradictory with $R>\frac{\sqrt{2} M_{R_{0}}}{\rho \varepsilon}$.

Then by Lemma 2.5 we know

$$
i\left(Q, \Omega_{R}, P\right)=1 .
$$

Now, by the additivity of fixed point index, combine (3.17) and (3.20) to conclude that

$$
i\left(Q, \Omega_{R} \backslash \bar{\Omega}_{r}, P\right)=i\left(Q, \Omega_{R}, P\right)-i\left(Q, \Omega_{r}, P\right)=1 .
$$

Therefore $Q$ has a fixed point in $\Omega_{R} \backslash \bar{\Omega}_{r}$, which is the positive solution of BVP (1.1).

Case (ii), since $\bar{f}_{0}<(1-L) \Gamma$, based on the definition of $\bar{f}_{0}$, we may choose $\varepsilon>0$ and $\omega>0$, so that

$$
f(t, u) \leq[(1-L) \Gamma-\varepsilon] u, \quad 0 \leq t \leq 1, \quad 0 \leq u \leq \omega .
$$

Let $r \in(0, \omega)$, we now prove that $\mu Q u \neq u$ for every $u \in \partial \Omega_{r}$, and $0<\mu \leq 1$. In fact, suppose the contrary, then there exist $u_{0} \in \partial \Omega_{r}$, and $0<\mu_{0} \leq 1$ such that $\mu_{0} Q u_{0}=u_{0}$. Let $\omega_{2}=A F u_{0}$, by (3.6), we have $u_{0}=\mu_{0} Q u_{0} \leq \frac{1}{1-L} A F u_{0} \leq \frac{1}{1-L} \omega_{2}$ and $\omega_{2}(t)$ satisfies BVP (2.4) with $h=F u_{0}$. Similarly to (3.18), we have

$$
\begin{aligned}
(1-L) \Gamma \int_{0}^{1} u_{0}(t) \sin \pi t d t & \leq \Gamma \int_{0}^{1} \omega_{2}(t) \sin \pi t d t=\int_{0}^{1} f\left(t, u_{0}(t)\right) \sin \pi t d t \\
& \leq[(1-L) \Gamma-\varepsilon] \int_{0}^{1} u_{0}(t) \sin \pi t d t .
\end{aligned}
$$

Since $\int_{0}^{1} u_{0}(t) \sin \pi t d t>0$, We see that $(1-L) \Gamma \leq(1-L) \Gamma-\varepsilon$, which is a contradiction. By Lemma 2.5, we have

$$
i\left(Q, \Omega_{r}, P\right)=1 \text {. }
$$

On the other hand, because $\underline{f}_{\infty}>\Gamma$, there exist $\varepsilon \in(0, \Gamma)$ and $H>0$ such that

$$
f(t, x) \geq(\Gamma+\varepsilon) x, \quad t \in[0,1], \quad x>H .
$$

Let $C=\max _{0 \leq t \leq 1,0 \leq x \leq H}|f(t, x)-(\Gamma+\varepsilon) x|+1$, then it is clear that

$$
f(t, x) \geq(\Gamma+\varepsilon) x-C, \quad t \in[0,1], \quad x \geq 0 .
$$

Choose $R>R_{0}=\max \{H / \rho, \omega\}, \forall u \in \partial \Omega_{R}$. By (3.13) and (3.25), we have

$$
u(s) \geq \rho\|u\|_{0}>H, \quad \forall s \in\left[\frac{1}{4}, \frac{3}{4}\right] .
$$

And so

$$
f(s, u(s)) \geq(\Gamma+\varepsilon) u(s) \geq(\Gamma+\varepsilon) \rho\|u\|_{0}, \quad \forall s \in\left[\frac{1}{4}, \frac{3}{4}\right] .
$$


From (3.6) and (3.26), we get

$$
\begin{aligned}
\|Q u\|_{0} \geq Q u\left(\frac{1}{2}\right)= & (H F u)\left(\frac{1}{2}\right) \geq(A F u)\left(\frac{1}{2}\right) \\
= & \int_{0}^{1} \int_{0}^{1} \int_{0}^{1} G_{1}\left(\frac{1}{2}, \delta\right) G_{2}(\delta, \tau) G_{3}(\tau, s) f(s, u(s)) d s d \tau d \delta \\
& \frac{3}{4} \frac{3}{4} \frac{3}{4} \frac{1}{4} \frac{1}{4} G_{1}\left(\frac{1}{2}, \delta\right) G_{2}(\delta, \tau) G_{3}(\tau, s)(\Gamma+\varepsilon) \rho\left\|_{0}\right\|_{0} d s d \tau d \delta \\
\geq & \int_{1} \int_{1} \frac{3}{4} \\
\geq & \delta_{1} \delta_{2} \delta_{3} m_{1} C_{12} C_{23}(\Gamma+\varepsilon) \rho\|u\|_{0} \int_{\frac{1}{4}} G_{3}(s, s) d s . \\
\geq & \frac{1}{2} \delta_{1} \delta_{2} \delta_{3} m_{1} m_{3} C_{12} C_{23}(\Gamma+\varepsilon) \rho\|u\|_{0}>0,
\end{aligned}
$$

from which we see that $\inf _{u \in \partial \Omega_{r}}\|Q u\|_{0}>0$, namely the hypotheses (i) of Lemma 2.6 holds. Next, we show that if $R$ is large enough, then $\mu Q u \neq u$ for any $u \in \partial \Omega_{R}$ and $\mu \geq 1$. In fact, suppose the contrary, then there exist $u_{0} \in \partial \Omega_{R}$ and $\mu_{0} \geq 1$ such that $\mu_{0} Q u_{0}=$ $u_{0}$, then by (3.6), $A F u_{0} \leq Q u_{0} \leq u_{0}=\mu_{0} Q u_{0} \leq \frac{\mu_{0}}{1-L} A F u_{0}$. Let $\omega_{0}=A F u_{0}$, then $\omega_{0} \leq u_{0} \leq \frac{\mu_{0}}{1-L} \omega_{0}$, and $\omega_{0}$ satisfies BVP (2.4), in which $h=F u_{0}$, consequently,

$$
\left\{\begin{array}{l}
-\omega_{0}^{(6)}-\gamma \omega_{0}^{(4)}+\beta \omega^{\prime \prime}{ }_{0}-\alpha \omega_{0}=f\left(t, u_{0}\right), \quad t \in[0,1] \\
\omega_{0}(0)=\omega_{0}(1)=\omega^{\prime \prime}{ }_{0}(0)=\omega^{\prime \prime}{ }_{0}(1)=\omega_{0}^{(4)}(0)=\omega_{0}^{(4)}(1)=0
\end{array}\right.
$$

After multiplying the two sides of the first equation in (3.27) by sin $\pi t$ and integrating on $[0,1]$, we have

$$
\begin{aligned}
\Gamma \int_{0}^{1} \omega_{0}(t) \sin \pi t d t & =\int_{0}^{1} f\left(t, u_{0}(t)\right) \sin \pi t d t \geq(\Gamma+\varepsilon) \int_{0}^{1} u_{0}(t) \sin \pi t d t-\frac{2 C}{\pi} \\
& \geq(\Gamma+\varepsilon) \int_{0}^{1} \omega_{0}(t) \sin \pi t d t-\frac{2 C}{\pi}
\end{aligned}
$$

Consequently, we obtain that

$$
\int_{0}^{1} \omega_{0}(t) \sin \pi t d t \leq \frac{2 C}{\pi \varepsilon}
$$

It's easy to prove that $\omega_{0}(t)$, the solution of LBVF (3.27) satisfies

$$
\omega_{0}(t) \geq \frac{\delta_{1} \delta_{2} \delta_{3} C_{12} C_{23}}{C_{1} C_{2} C_{3} M_{1} M_{2}} G_{1}(t, t)\left\|\omega_{0}\right\|_{0},
$$

and accordingly,

$$
\int_{0}^{1} \omega_{0}(t) \sin \pi t d t \geq \frac{\delta_{1} \delta_{2} \delta_{3} C_{12} C_{23}\left\|\omega_{0}\right\|_{0}}{C_{1} C_{2} C_{3} M_{1} M_{2}} \int_{0}^{1} G_{1}(t, t) \sin \pi t d t,
$$


by (3.28), we get

$$
\left\|\omega_{0}\right\|_{0} \leq \frac{2 C C_{1} C_{2} C_{3} M_{1} M_{2}}{\delta_{1} \delta_{2} \delta_{3} C_{12} C_{23} \pi \varepsilon}\left(\int_{0}^{1} G_{1}(t, t) \sin \pi t d t\right)^{-1}:=\bar{R},
$$

Consequently, $\left\|u_{0}\right\|_{0} \leq \frac{\mu_{0}}{1-L}\left\|\omega_{0}\right\|_{0} \leq \frac{\mu_{0}}{1-L} \bar{R}$.

We choose $R>\max \left\{\frac{\mu_{0}}{1-L} \bar{R}, R_{0}\right\}$, then to any $u \in \partial \Omega_{R}, \mu \geq 1$, there is always $\mu Q u \neq u$. Hence, hypothesis (ii) of Lemma 2.6 also holds. By Lemma 2.6, we have

$$
i\left(Q, \Omega_{R}, P\right)=0 .
$$

Now, by the additivity of fixed point index, combine (3.23) and (3.31) to conclude that

$$
i\left(Q, \Omega_{R} \backslash \bar{\Omega}_{r}, P\right)=i\left(Q, \Omega_{R}, P\right)-i\left(Q, \Omega_{r}, P\right)=-1
$$

Therefore, $Q$ has a fixed poind in $\Omega_{R} \backslash \bar{\Omega}_{r}$, which is the positive solution of BVP (1.1). The proof is completed.

From Theorem 3.1, we immediately obtain the following.

Corollary 3.1. Assume $\left(H_{1}\right)-\left(H_{3}\right)$ hold, then in each of the following cases:

(i) $\underline{f}_{-0}=\infty, \bar{f}_{\infty}=0$, (ii) $\bar{f}_{0}=0, \underline{f}_{-}=\infty$,

the BVP (1.1) has at least one positive solution.

\section{Multiple solutions}

Next, we study the multiplicity of positive solutions of BVP (1.1) and assume in this section that

$\left(H_{4}\right)$ there is a $p>0$ such that $0 \leq u \leq p$ and $0 \leq t \leq 1$ imply $f(t, u)<\eta p$, where $\eta=\left(\frac{C_{1} C_{2} C_{3} M_{1} M_{2}}{1-L} \int_{0}^{1} G_{1}(s, s) d s\right)^{-1}$.

$\left(H_{5}\right)$ there is a $p>0$ such that $\sigma p \leq u \leq p$ and $0 \leq t \leq 1$ imply $f(t, u) \geq \lambda p$, where $\lambda^{-1}=\delta_{1} \delta_{2} \delta_{3} m_{1} C_{12} C_{23} \int_{\frac{1}{4}}^{\frac{3}{4}} G_{3}(s, s) d s$. Here, $\sigma$ can be defined as (3.10).

Theorem 4.1. Assume $\left(H_{1}\right)-\left(H_{4}\right)$ hold. If $f_{-0}>\Gamma$ and $f_{-\infty}>\Gamma$, then BVP (1.1) has at least two positive solution $u_{1}$ and $u_{2}$ such that $0 \leq\left\|u_{1}\right\|_{0} \leq p \leq\left\|u_{2}\right\|_{0}$.

Proof. According to the proof of Theorem 3.1, there exists $0<r_{0}<p<R_{1}<+\infty$, such that $0<r<r_{0}$ implies $i\left(Q, \Omega_{r}, P\right)=0$ and $R \geq R_{1}$ implies $i\left(Q, \Omega_{R}, P\right)=0$.

Next we prove $i\left(Q, \Omega_{p}, P\right)=1$ if $\left(H_{4}\right)$ is satisfied. In fact, for every $u \in \partial \Omega_{p}$, based on the preceding definition of $Q$ we come to

$$
\begin{aligned}
(Q u)(t) & =(H F u)(t) \leq \frac{1}{1-L}\|A F u\|_{0} \\
& =\frac{1}{1-L} \max _{0 \leq t \leq 1}\left|\int_{0}^{1} \int_{0}^{1} \int_{0}^{1} G_{1}(t, \delta) G_{2}(\delta, \tau) G_{3}(\tau, s)(F u)(s) d s d \tau d \delta\right| \\
& \leq \frac{C_{1} C_{2} C_{3} M_{1} M_{2}}{1-L} \mid \int_{0}^{1} G_{3}(s, s) f(s, u(s) d s \mid
\end{aligned}
$$


Consequently,

$$
\begin{aligned}
\|Q u\|_{0} & \leq \frac{C_{1} C_{2} C_{3} M_{1} M_{2}}{1-L}\left|\int_{0}^{1} G_{3}(s, s) f(s, u(s)) d s\right| \\
& \leq \frac{C_{1} C_{2} C_{3} M_{1} M_{2}}{1-L} \int_{0}^{1} G_{3}(s, s) \eta p d s=p=\|u\|_{0} .
\end{aligned}
$$

Therefore, by (ii) of Lemma 2.7 we have

$$
i\left(Q, \Omega_{p}, P\right)=1 \text {. }
$$

Combined with (3.17), (3.31), and (4.1), we have

$$
\begin{aligned}
& i\left(Q, \Omega_{R} \backslash \bar{\Omega}_{p}, P\right)=i\left(Q, \Omega_{R}, P\right)-i\left(Q, \Omega_{p}, P\right)=-1 . \\
& i\left(Q, \Omega_{p} \backslash \bar{\Omega}_{r}, P\right)=i\left(Q, \Omega_{p}, P\right)-i\left(Q, \Omega_{r}, P\right)=1 .
\end{aligned}
$$

Therefore, $Q$ has fixed points $u_{1}$ and $u_{2}$ in $\Omega_{p} \backslash \bar{\Omega}_{r}$ and $\Omega_{R} \backslash \bar{\Omega}_{p}$, respectively, which means that $u_{1}(t)$ and $u_{2}(t)$ are positive solutions of BVP (1.1) and $0 \leq\left\|u_{1}\right\|_{0} \leq p \leq \|$ $u_{2} \|_{0}$. The proof is completed.

Theorem 4.2. Assume $\left(H_{1}\right)-\left(H_{3}\right)$ and $\left(H_{5}\right)$ can be established, and $\bar{f}_{0}<(1-L) \Gamma$ and $\bar{f}_{\infty}<(1-L) \Gamma$, then BVP (1.1) has at least two positive solution $u_{1}$ and $u_{2}$ such that 0 $\leq\left\|u_{1}\right\|_{0} \leq p \leq\left\|u_{2}\right\|_{0}$.

Proof. According to the proof of Theorem 3.1, there exists $0<\omega<p<R_{2}<+\infty$, such that $0<r<\omega$ implies $i\left(Q, \Omega_{r}, P\right)=1$ and $R \geq R_{2}$ implies $i\left(Q, \Omega_{R}, P\right)=1$.

We now prove that $i\left(Q, \Omega_{p}, P\right)=0$ if $\left(H_{5}\right)$ is satisfied. In fact, for every $u \in \partial \Omega_{p}$, by (3.13) we come to $\rho p \leq \rho\|u\|_{0} \leq u(t) \leq\|u\|_{0}=p, t \in[1 / 4,3 / 4]$, accordingly, by $\left(H_{5}\right)$, we have

$$
f(t, u) \geq \lambda p, \quad t \in\left[\frac{1}{4}, \frac{3}{4}\right], \quad \forall u \in \partial \Omega_{p} .
$$

from the proof of (ii) of Theorem 3.1, we have

$$
\begin{aligned}
\|Q u\|_{0} \geq & Q u\left(\frac{1}{2}\right)=(H F u)\left(\frac{1}{2}\right) \geq(A F u)\left(\frac{1}{2}\right) \\
= & \int_{0}^{1} \int_{0}^{1} \int_{0}^{1} G_{1}\left(\frac{1}{2}, \delta\right) G_{2}(\delta, \tau) G_{3}(\tau, s) f(s, u(s)) d s d \tau d \delta \\
& \frac{1}{4} \int_{1}^{\frac{3}{4}} \frac{1}{4} \int_{\frac{1}{4}}^{\frac{3}{4}} G_{1}\left(\frac{1}{2}, \delta\right) G_{2}(\delta, \tau) G_{3}(\tau, s) \lambda p d s d \tau d \delta \\
\geq & \delta_{1} \delta_{2} \delta_{3} m_{1} C_{12} C_{23} \int_{\frac{1}{4}}^{\frac{3}{4}} G_{3}(s, s) \lambda p d s=p=\|u\|_{0} .
\end{aligned}
$$


Therefore, $\|Q u\|_{0} \geq Q u\left(\frac{1}{2}\right) \geq\|u\|_{0}$, according to (i) of Lemma 2.7, we come to

$$
i\left(Q, \Omega_{p}, P\right)=0
$$

Combined with (3.20), (3.23), and (4.2), there exist

$$
\begin{aligned}
& i\left(Q, \Omega_{R} \backslash \bar{\Omega}_{p}, P\right)=i\left(Q, \Omega_{R}, P\right)-i\left(Q, \Omega_{p}, P\right)=1 . \\
& i\left(Q, \Omega_{p} \backslash \bar{\Omega}_{r}, P\right)=i\left(Q, \Omega_{p}, P\right)-i\left(Q, \Omega_{r}, P\right)=-1 .
\end{aligned}
$$

Therefore, $Q$ has fixed points $u_{1}$ and $u_{2}$ in $\Omega_{p} \backslash \bar{\Omega}_{r}$ and $\Omega_{R} \backslash \bar{\Omega}_{p}$, respectively, which means that $u_{1}(t)$ and $u_{2}(t)$ are positive solutions of BVP (1.1) and $0 \leq\left\|u_{1}\right\|_{0} \leq p \leq \|$ $u_{2} \|_{0}$. The proof is completed.

Theorem 4.3. Assume that $\left(H_{1}\right)-\left(H_{3}\right)$ hold. If $\underline{f}_{0}>\Gamma$ and $\bar{f}_{\infty}<(1-L) \Gamma$, and there exists $p_{2}>p_{1}>0$ that satisfies

(i) $f(t, u)<\eta p_{1}$ if $0 \leq t \leq 1$ and $0 \leq u \leq p_{1}$,

(ii) $f(t, u) \geq \lambda p_{2}$ if $0 \leq t \leq 1$ and $\sigma p_{2} \leq u \leq p_{2}$,

where $\eta, \sigma, \lambda$ are just as the above, then BVP (1.1) has at least three positive solutions $u_{1}, u_{2}$, and $u_{3}$ such that $0 \leq\left\|u_{1}\right\|_{0} \leq p_{1} \leq\left\|u_{2}\right\|_{0} \leq p_{2} \leq\left\|u_{3}\right\|_{0}$.

Proof. According to the proof of Theorem 3.1, there exists $0<r_{0}<p_{1}<p_{2}<R_{3}<$ $+\infty$, such that $0<r<r_{0}$ implies $i\left(Q, \Omega_{r}, P\right)=0$ and $R \geq R_{3}$ implies $i\left(Q, \Omega_{R}, P\right)=1$.

From the proof of Theorems 4.1 and 4.2 , we have $i\left(Q, \Omega_{p_{1}}, P\right)=1, i\left(Q, \Omega_{p_{2}}, P\right)=0$. Combining the four afore-mentioned equations, we have

$$
\begin{aligned}
& i\left(Q, \Omega_{R} \backslash \bar{\Omega}_{p_{2}}, P\right)=i\left(Q, \Omega_{R}, P\right)-i\left(Q, \Omega_{p_{2}}, P\right)=1 . \\
& i\left(Q, \Omega_{p_{2}} \backslash \bar{\Omega}_{p_{1}}, P\right)=i\left(Q, \Omega_{p_{2}}, P\right)-i\left(Q, \Omega_{p_{1}}, P\right)=-1 . \\
& i\left(Q, \Omega_{p_{1}} \backslash \bar{\Omega}_{r}, P\right)=i\left(Q, \Omega_{p_{1}}, P\right)-i\left(Q, \Omega_{r}, P\right)=1 .
\end{aligned}
$$

Therefore, $Q$ has fixed points $u_{1}, u_{2}$ and $u_{3}$ in $\Omega_{R} \backslash \bar{\Omega}_{p_{2}}, \quad \Omega_{p_{2}} \backslash \bar{\Omega}_{p_{1}}$ and $\Omega_{p_{1}} \backslash \bar{\Omega}_{r}$, respectively, which means that $u_{1}(t), u_{2}(t)$ and $u_{3}(t)$ are positive solutions of BVP (1.1) and $0 \leq\left\|u_{1}\right\|_{0} \leq p_{1} \leq\left\|u_{2}\right\|_{0} \leq p_{2} \leq\left\|u_{3}\right\|_{0}$. The proof is completed.

\section{Acknowledgements}

The author is very grateful to the anonymous referees for their valuable suggestions, and to be sponsored by the Tutorial Scientific Research Program Foundation of Education Department of Gansu Province P.R.China(1110-05).

\section{Authors' contributions}

WL conceived of the study, and participated in its design and coordination. The author read and approved the final manuscript.

\section{Competing interests}

The author declares that they have no competing interests.

Received: 22 November 2011 Accepted: 22 February 2012 Published: 22 February 2012

\section{References}

1. Tersian, S, Chaparova, J: Periodic and homoclinic solutions of some semilinear sixth-order ODE. J Math Anal Appl. 272, 223-239 (2002). doi:10.1016/S0022-247X(02)00153-1

2. Gardner, RA, Jones, CKRT: Traveling waves of a perturbed diffusion equation arising in a phase field model. Indiana Univ Math J. 39(4):1197-1222 (1990). doi:10.1512/iumj.1990.39.39054

3. Caginalp, G, Fife, P: Higher order phase field models and detailed anisotropy. Phys Rev B. 34, 4940-4943 (1986). doi:10.1103/PhysRevB.34.4940

4. Gyulov, T, Morosanu, G, Tersian, S: Existence for a semilinear sixth-order ODE. J Math Anal Appl. 321, 86-98 (2006). doi:10.1016/j.jmaa.2005.08.007

5. Zhang, L, An, Y: Existence and multiplicity of positive solutions of a boundary-value problem for sixth-order ode with three parameters. Bound Value Probl 2010, 13 (2010). (Article ID 878131) doi:10.1155/2010/878131 
6. Bates, P, Fife, P, Gardner, R, Jones, CKRJ: The existence of traveling wave solutions of a generalized phase-field model. SIAM J Math Anal. 28, 60-93 (1997). doi:10.1137/S0036141095283820

7. Peletier, LA, Troy, WC, Van der Vorst, RCAM: Stationary solutions of a fourth-order nonlinear diffusion equation. Diff Equ. $31,301-314(1995)$

8. Chai, G: Existence of positive solutions for fourth-order boundary value problem with variable parameters. Nonlinear Anal. 66, 870-880 (2007). doi:10.1016/j.na.2005.12.028

9. Zhang, $M$, Wei, Z: Existence of positive solutions for fourth-order $m$-point boundary value problem with variable parameters. Appl Math Comput. 190, 1417-1431 (2007). doi:10.1016/j.amc.2007.02.019

10. Li, Y: Positive solutions of fourth-order boundary value problems with two parameters. J Math Anal Appl. 281, 477-484 (2003). doi:10.1016/50022-247X(03)00131-8

11. Guo, D, Lakshmikantham, V, Liu, X: Nonlinear Integral Equtions in Abstract Spaces. Kluwer Academic Publishers, Dordrecht-Boston-Loudon (1996)

12. Guo, D, Lakshmikantham, V: Nonlinear Problems in Abstract Cones. Academic Press, New York (1998)

doi:10.1186/1687-2770-2012-22

Cite this article as: Li: The existence and multiplicity of positive solutions of nonlinear sixth-order boundary value problem with three variable coefficients. Boundary Value Problems 2012 2012:22.

\section{Submit your manuscript to a SpringerOpen ${ }^{\circ}$} journal and benefit from:

- Convenient online submission

- Rigorous peer review

- Immediate publication on acceptance

- Open access: articles freely available online

- High visibility within the field

- Retaining the copyright to your article

Submit your next manuscript at $\gg$ springeropen.com 